\title{
Ultrasound-induced microbubble cavitation via a transcanal or transcranial approach facilitates inner ear drug delivery
}

\author{
Ai-Ho Liao, ${ }^{1,2}$ Chih-Hung Wang, ${ }^{3,4,5}$ Ping-Yu Weng, ${ }^{6}$ Yi-Chun Lin, ${ }^{3}$ Hao Wang, ${ }^{4}$ Hang-Kang Chen, ${ }^{3}$ \\ Hao-Li Liu, ${ }^{7}$ Ho-Chiao Chuang, ${ }^{6}$ and Cheng-Ping Shih ${ }^{4}$ \\ ${ }^{1}$ Graduate Institute of Biomedical Engineering, National Taiwan University of Science and Technology, Taipei, Taiwan. \\ 2Department of Biomedical Engineering and ${ }^{3} \mathrm{G}$ raduate Institute of Medical Sciences, National Defense Medical Center, \\ Taipei, Taiwan. ${ }^{4}$ Department of Otolaryngology-Head and Neck Surgery, Tri-Service General Hospital, National Defense \\ Medical Center, Taipei, Taiwan. ${ }^{5}$ Taichung Armed Forces General Hospital, Taichung, Taiwan. ${ }^{6}$ Department of Mechanical \\ Engineering, National Taipei University of Technology, Taipei, Taiwan. 'Department of Electrical Engineering, Chang Gung \\ University, Tao-Yuan, Taiwan.
}

Ultrasound-induced microbubble (USMB) cavitation is widely used to promote drug delivery. Our previous study investigated USMB targeting the round window membrane by applying the ultrasound transducer to the tympanic bulla. In the present study, we further extended the use of this technology to enhance drug delivery to the inner ear by introducing the ultrasound transducer into the external auditory canal (EAC) or applying it to the skull. Using a 3-dimensional-printed diffusion apparatus mimicking the pathway for ultrasound passing through and reaching the middle ear cavity in vitro, the models simulating the transcanal and transcranial approach demonstrated 4.8-fold- and 3.7-fold-higher delivery efficiencies, respectively. In an in vivo model of guinea pigs, by filling tympanic bulla with microbubbles and biotin-FITC, USMB applied transcanally and transcranially induced 2.8-fold and 1.5-fold increases in biotin-FITC delivery efficiencies, respectively. In addition, the gentamicin uptake by cochlear and vestibular hair cells and gentamicin-induced hair cell loss were significantly enhanced following transcanal application of USMB. On the 28th day after transcanal USMB, safety assessment showed no significant changes in the hearing thresholds and the integrity of cochlea. These are the first results to our knowledge to demonstrate the feasibility and support the potential clinical application of applying USMB via EAC to facilitate drug delivery into the inner ear.

Authorship note: AHL and CHW contributed equally to this work.

Conflict of interest: The authors have declared that no conflict of interest exists.

Copyright: (c) 2020, American Society for Clinical Investigation.

Submitted: August 20, 2019

Accepted: December 26, 2019

Published: February 13, 2020

Reference information: JCI Insight. 2020;5(3):e132880.

https://doi.org/10.1172/jci.

insight.132880.

\section{Introduction}

The clinical administration of drugs to the inner ear can be performed systemically or locally (1). The local intratympanic injection of drugs has generally been used since 1990 and is recognized as the most effective and least harmful current method. The treatment agent is injected into the middle ear cavity via an intratympanic injection, and it enters the inner ear via absorption by the round window membrane (RWM) and oval window $(2,3)$. The distribution of the drug in the inner ear depends on which window it enters the inner ear through and whether it is administered systemically or locally. The perilymph circulation in the inner ear is slow, and the blood-labyrinth barrier prevents a drug in the blood from entering the inner ear; both mechanisms make the systemic route for inner ear drug delivery inefficient (1). Moreover, the drug will accumulate in cochlea at only a low concentration; therefore, many methods are currently being investigated to improve the permeability of the RWM for local drug delivery. Some efficient carriers such as poly (lactic-co-glycolic acid) nanoparticles and phospholipid-based nanoparticles are used as inner ear nanoparticulate carriers to produce better therapeutic effects and to maintain long-term particle circulation in the perilymph $(4,5)$.

Some agents such as collagenase (6), histamine and local anesthetic phenols (7), and hyaluronic acid (8) have been applied to the RWM to destroy its outer epithelial cells and thereby enhance drug delivery without resorting to surgery. However, these approaches may cause infection in the middle ear cavity and damage the RWM - or even the inner ear, itself (9). Therefore, identifying a simple and effective method that does not damage the structure of the cochlea is an important issue for inner ear drug delivery. A previous in vitro study 
created microperforations in the RWM with a $12.5-\mu \mathrm{m}$-diameter needle to enhance diffusion through the membrane (10). Two designs of multiple (4 or 8 ) serrated needles were investigated, and it was found that an octagonal needle arrangement produced an oval-shaped perforation of the RWM that performed better than when using 4 needles (11). However, the ability to create a hole of an appropriate size in the RWM during surgical manipulation without a deleterious increase in the intracochlear pressure remains to be demonstrated.

Improved versions of the microbubbles (MBs) that are commonly used as ultrasound (US) contrast agents have recently be used to improve the diagnostic confidence in echography and in combination with US as a tool for drug delivery and therapeutic monitoring. Applying this technique to clinical use requires evaluations of the sonoporation mechanisms, US parameters, and drug types and doses (12). Transcranial noninvasive MRI-guided focused US is a method of disrupting the blood-brain barrier (BBB) for enhancing brain tumor treatments via i.v. injected MBs interacting with the US (13). Additionally, MB echoes can be detected with passive cavitation detection (PCD) for real-time feedback control the BBB disruption during focused US-induced MB (USMB) cavitation $(14,15)$. The use of focused USMB cavitation for BBB disruption is receiving considerable attention, and there are now widely accepted measures of safety and a good understanding of its clinical relevance (16). However, the feasibility of this noninvasive technique has not been widely investigated for other applications.

Our previous study demonstrated the practical application of using USMB to increase the permeability of the RWM, in which a surgical technique was used to remove the temporal bone and expose the membrane (17). We subsequently expanded that technique to explore the delivery of dexamethasone mediated by USMB and its protective effects against noise exposure in animal models (18). In clinical practice, the transcanal approach is often indicated for transtympanic injections of drugs to treat various inner ear diseases and is applied to most patients undergoing endoscopic ear surgery (19). The transcanal approach has the advantage of conveniently and directly reaching the middle ear without needing to make an external incision.

The present study used 2 nonsurgical techniques (transcanal and transcranial USMB approaches) to facilitate drug delivery into the inner ear through the RWM in vivo. Before performing the animal experiments, the feasibilities of the USMB techniques for transcanal and transcranial approaches were demonstrated in vitro with a 3-dimensional-printed (3D-printed) diffusion apparatus that mimics 2 pathways for the US sonication: (a) through the external auditory canal and tympanic membrane, reaching the middle ear cavity and targeting the RWM, and (b) through the skull bone, reaching the middle ear cavity and targeting the RWM.

For measuring drug diffusion through the guinea pig RWM, some previous studies have used 3D-printed acrylic holders adapted to the round window niche using 3-mL Franz diffusion cells $(10,11)$. However, the large volume of these Franz cells and the time-consuming procedures necessary to obtain transport parameters greatly restrict their application. A previous study demonstrated a 3D-printed, pocket-size diffusion cell as an in vitro tool for analyzing skin permeation (20). Therefore, a potentially new 3D-printed diffusion apparatus for the initial assessment of drug permeation through the RWM was necessary to simulate the volume of the middle ear cavity, which might affect the efficacy of USMB cavitation for inner ear drug delivery. Moreover, PCD was used to evaluate the use of stable or inertial USMB cavitation for inner ear drug delivery. Since the transcanal and transcranial delivery methods are noninvasive, it is expected to be straightforward to translate USMB cavitation to the clinical enhancement of inner ear drug delivery in the near future.

\section{Results}

Optimizing the in vivo parameters for middle ear USMB. High-frequency US images of $1.4 \times 10^{7} \mathrm{MBs} / \mathrm{mL}$ albumin-shelled MBs without and with US sonication at $1,2,3$, and $4 \mathrm{~W} / \mathrm{cm}^{2}$ for 1 minute are shown in Figure 1 , A-E, respectively; the signal intensities in Figure 1, A-E, are quantified in Figure 1F. The image intensity of the MBs before US sonication was $21.1 \pm 0.2 \mathrm{~dB}$, and $7.5 \pm 0.9,4.9 \pm 0.9,3.8 \pm 0.3$, and $3.8 \pm 0.4 \mathrm{~dB}$ with US sonication at 1, 2, 3, and $4 \mathrm{~W} / \mathrm{cm}^{2}$ for 1 minute, respectively. The efficiencies of $\mathrm{MB}$ destruction for US sonication at power densities of $1,2,3$, and $4 \mathrm{~W} / \mathrm{cm}^{2}$ for 30 seconds were $64.45 \%, 76.78 \%, 81.99 \%$, and $81.99 \%$, respectively. The temperature in the chamber during sonication at the different US powers increased by $1.25^{\circ} \mathrm{C}-1.80^{\circ} \mathrm{C}$. In this experiment, applying US at a power density of $3 \mathrm{~W} / \mathrm{cm}^{2}$ for 1 minute almost destroyed the MBs, and this was the highest efficiency setting for the subsequent in vivo transcanal and transcranial approaches.

High-frequency US images of MBs without and with US sonication at 1, 2, 3, and $4 \mathrm{~W} / \mathrm{cm}^{2}$ for 3 minutes through the skull bone are shown in Figure 2, A-E, respectively; the corresponding image intensities of the 

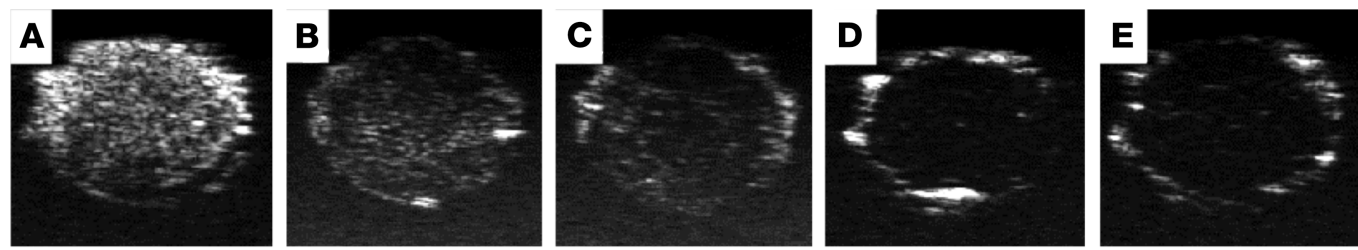

$\mathbf{F}$

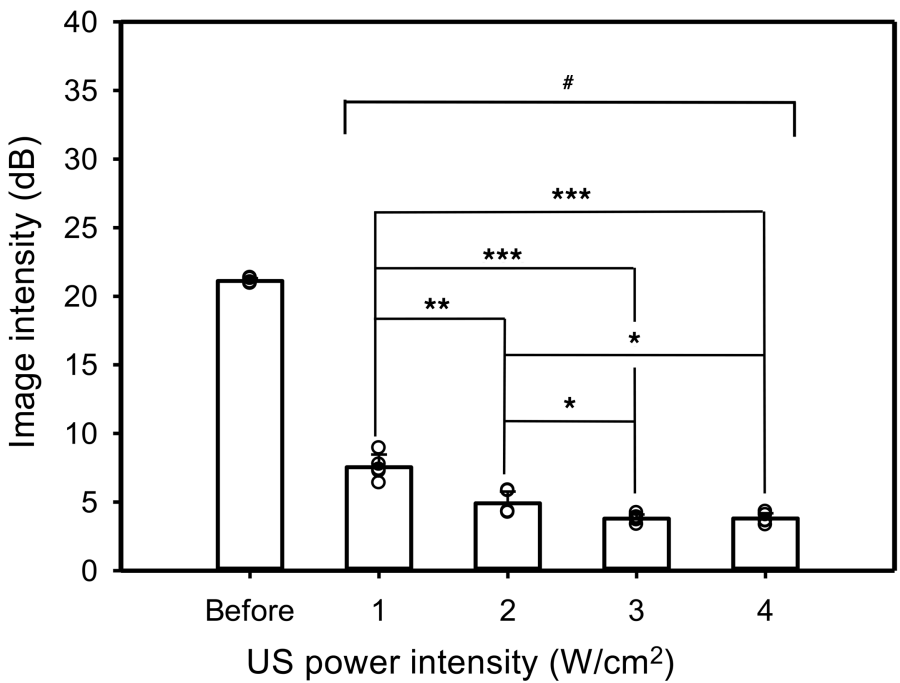

Figure 1. High-frequency US images of MBs before and after US sonication. (A-E) Images were obtained before application of US (A) and following application of US at power densities of 1 (B), 2 (C), 3 (D), and 4 (E) W/cm² for 1 minute. Diameter of round areas in the images $4 \mathrm{~mm}$. (F) Quantification of the signal intensities. Multigroup comparisons using 1-way ANOVA, followed by post hoc Tukey's tests. Values are expressed as mean $\pm \operatorname{SD}(n=5$ per group). US, ultrasound; MBs, microbubbles. ${ }^{*} P<0.05$, ${ }^{* *} P<0.01,{ }^{* * *} P<0.001,{ }^{\#} P<0.001$ relative to control group; 1-way ANOVA followed by Tukey's multiple-comparisons test and 2-tailed Student's $t$ test.

MBs were $20.1 \pm 0.9,8.6 \pm 1.5,6.0 \pm 0.6,4.1 \pm 0.2$, and $4.2 \pm 0.6 \mathrm{~dB}$, respectively. The efficiencies of MB destruction for US sonication at power densities of $1,2,3$, and $4 \mathrm{~W} / \mathrm{cm}^{2}$ for 3 minutes were $57.20 \%, 70.15 \%$, $79.60 \%$, and $79.10 \%$, respectively. The temperatures on the skull bone and in the chamber of the phantom during US sonication at the different powers increased by $3.90^{\circ} \mathrm{C}-8.80^{\circ} \mathrm{C}$ and $1.10^{\circ} \mathrm{C}-2.30^{\circ} \mathrm{C}$, respectively. The results indicate that the albumin-shelled MBs were significantly destroyed after being sonicated with US at $3 \mathrm{~W} / \mathrm{cm}^{2}$ through either the tympanic membrane or the skull bone $(P<0.001$, Figure $1 \mathrm{~F} ; P<0.001$, Figure $2 \mathrm{~F}$ ). Therefore, the highest US power density of $3 \mathrm{~W} / \mathrm{cm}^{2}$ (for 1 and 3 minutes in the transcanal and transcranial approaches, respectively) was used in the subsequent in vitro and in vivo experiments.

In vitro cavitation detection. In vitro tube-phantom experiments were conducted to characterize how the energy spectrum density changed with $\mathrm{MB}$ infusion. Figure 3 shows the area under the receiver operating characteristics curve (AUC) for $2 \mathrm{MB}$ concentrations (1, original, $1.40 \times 10^{8} \mathrm{MBs} / \mathrm{mL} ; 10 \times$, 10 -fold dilution $1.40 \times 10^{7} \mathrm{MBs} / \mathrm{mL}$ ) for 120 seconds (60-180 seconds). The detected AUC increased with the injections of MBs at the 60th second and returned to the baseline value when the injection of MBs into the tube phantom was stopped after 180 seconds. In Figure 3A, the AUCs for the inertial cavitation and stable cavitation of MBs at the $10 \times$ and $1 \times$ dilutions from $60-180$ seconds were $3102 \pm 899$ (inertial cavitation, MBs $10 \times$ ), $1449 \pm 340$ (inertial cavitation, MBs $1 \times$ ), $1263 \pm 323$ (stable cavitation, MBs 10×), and $641 \pm 145$ (stable cavitation, MBs $1 \times)$. In Figure 3B, with US sonication through the skull bone, the detected AUCs for the inertial cavitation and stable cavitation of MBs at the $10 \times$ and $1 \times$ dilutions from 60-180 seconds were $2292 \pm 555$ (inertial cavitation, MBs 10×), $2006 \pm 220$ (inertial cavitation, MBs 1×), $960 \pm 242$ (stable cavitation, MBs 10×), and 796 \pm 88 (stable cavitation, MBs $1 \times$ ). US sonication at a power density of $3 \mathrm{~W} / \mathrm{cm}^{2}$ resulted in inertial cavitation that was more significant than stable cavitation at a dilution of either $10 \times$ or $1 \times$, especially after injecting MBs. When the US sonication was applied through the skull bone, the detected average AUCs decreased in all experimental groups due to the attenuation caused by the bone. Moreover, the inertial cavitation was highest for MBs at the $10 \times$ dilution, and so this was used in the subsequent in vitro and in vivo experiments. 

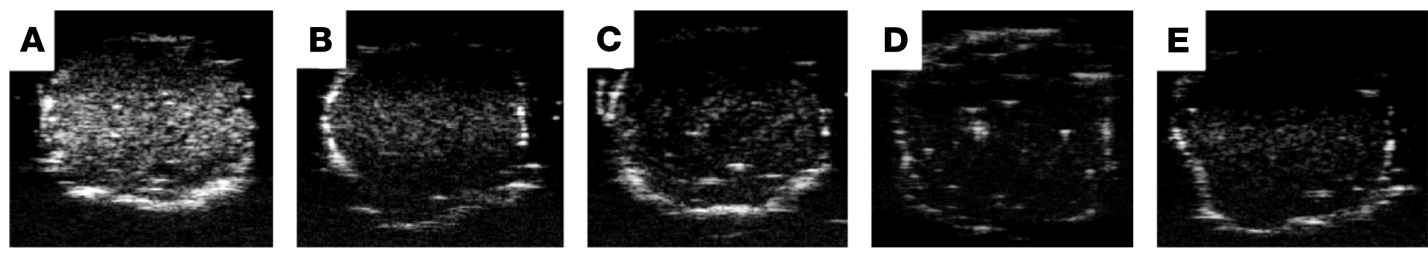

$\mathbf{F}$

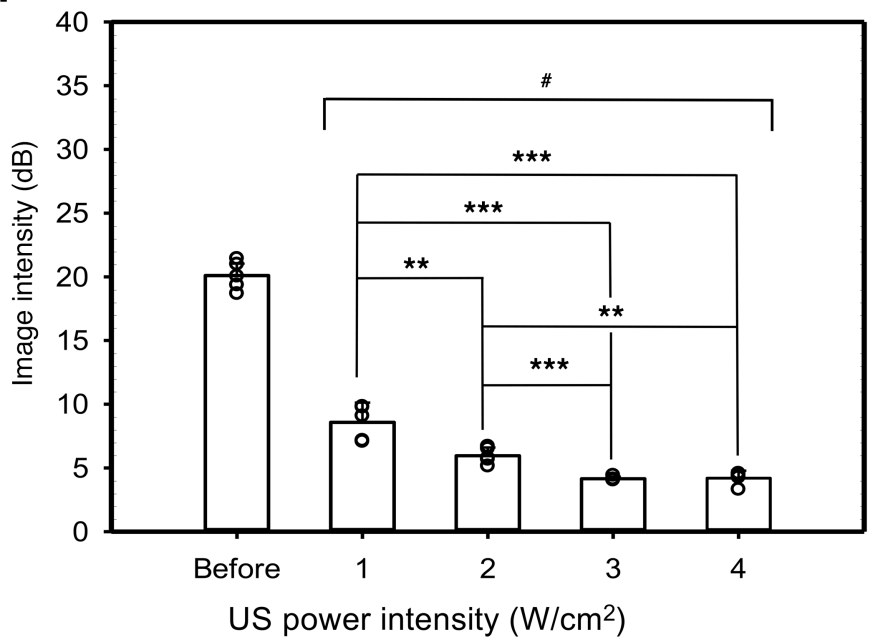

Figure 2. High-frequency US images of MBs before and after US sonication through the skull base. (A-E) Images were obtained before US (A) and following application of US at power densities of 1 (B), 2 (C), 3 (D), and 4 (E) W/ $\mathrm{cm}^{2}$ for 3 minutes. The diameter of the round area in the images is $4 \mathrm{~mm}$. (F) Quantification of the signal intensities. Multigroup comparisons using 1-way ANOVA, followed by post hoc Tukey's tests. Values are expressed as mean \pm SD ( $n=5$ per group). ${ }^{*} P<0.05$, ${ }^{*} P<0.01,{ }^{* * *} P<0.001$, ${ }^{*} P<0.001$ relative to control group; 1 -way ANOVA followed by Tukey's multiple-comparisons test and 2-tailed Student's $t$ test.

In vitro efficacy of USMBs for RWM permeation. Figure 4A shows the biotin-FITC concentrations in the 4 groups for membrane penetration as analyzed using a fluorometer. In the $3 \mathrm{D}$-printed diffusion cell model, the USMB cavitation greatly improved the biotin-FITC delivery in groups M1 (the single-membrane model), M1-20 (the single-membrane model, simulating the deviating the US transducer $20^{\circ}$ from the center), M2 (the 2-membranes model, simulating the transcanal approach), and $\mathrm{M} 1+\mathrm{B}$ (the single-membrane model through the skull bone, simulating the transcranial approach) by about 6.9, 5.7, 4.8, and 3.7 times, respectively, compared with solely soaking biotin-FITC within the diffusion cell. The concentration differed significantly $(P<0.05)$ between the round window soaking (RWS) and USM (USMB treatments) groups.

Transcanal or transcranial USMB promote the delivery of drugs into the inner ear without auditory damage. To confirm the efficiency of in vivo USMB-mediated drug delivery to the inner ear system, biotin-FITC was used as the delivery agent in the guinea pig model. The results for the inner ear perilymph in the transcanal and transcranial arms are shown in Figure 4B. In the transcanal arm, the fluorescence intensity of biotin-FITC when using the transcanal approach was significantly higher in the USM group than in the RWS group $(4620 \pm 202.9$ vs. $1633 \pm 72.6, P=0.013)$. When performing the transcranial approach, the USM group also exhibited a higher level of biotin-FITC than that in the RWS group $(10,113 \pm 338.8$ vs. 6700.0 $\pm 69.7, P=0.049$ ). The application of USMBs increased the delivery of biotin-FITC by approximately 2.8 fold in the transcanal approach and 1.5-fold in the transcranial approach. These findings demonstrate that exposure to USMB via either of the 2 approaches facilitated the delivery of drugs into the inner ear and that this was higher in the transcanal approach than the transcranial approach.

Since the transcanal approach is also a more practical procedure for clinical application, the transcanal USM group was assessed in the following experiments. Gentamicin was used to further assess the efficacy of using transcanal USMB to enhance drug delivery to the inner ear. In the clinical situation, gentamicin can be injected intratympanically to treat patients with Ménière's disease to improve the episodes of vertigo that they experience (21). In the USM group of the present experiments, $200 \mu \mathrm{L}$ of a mixture of gentamicin $(40 \mu \mathrm{g} / \mathrm{mL})$ and MBs $\left(1.40 \times 10^{7} \mathrm{MBs} / \mathrm{mL}\right)$ were injected into the middle ear cavity; then, 

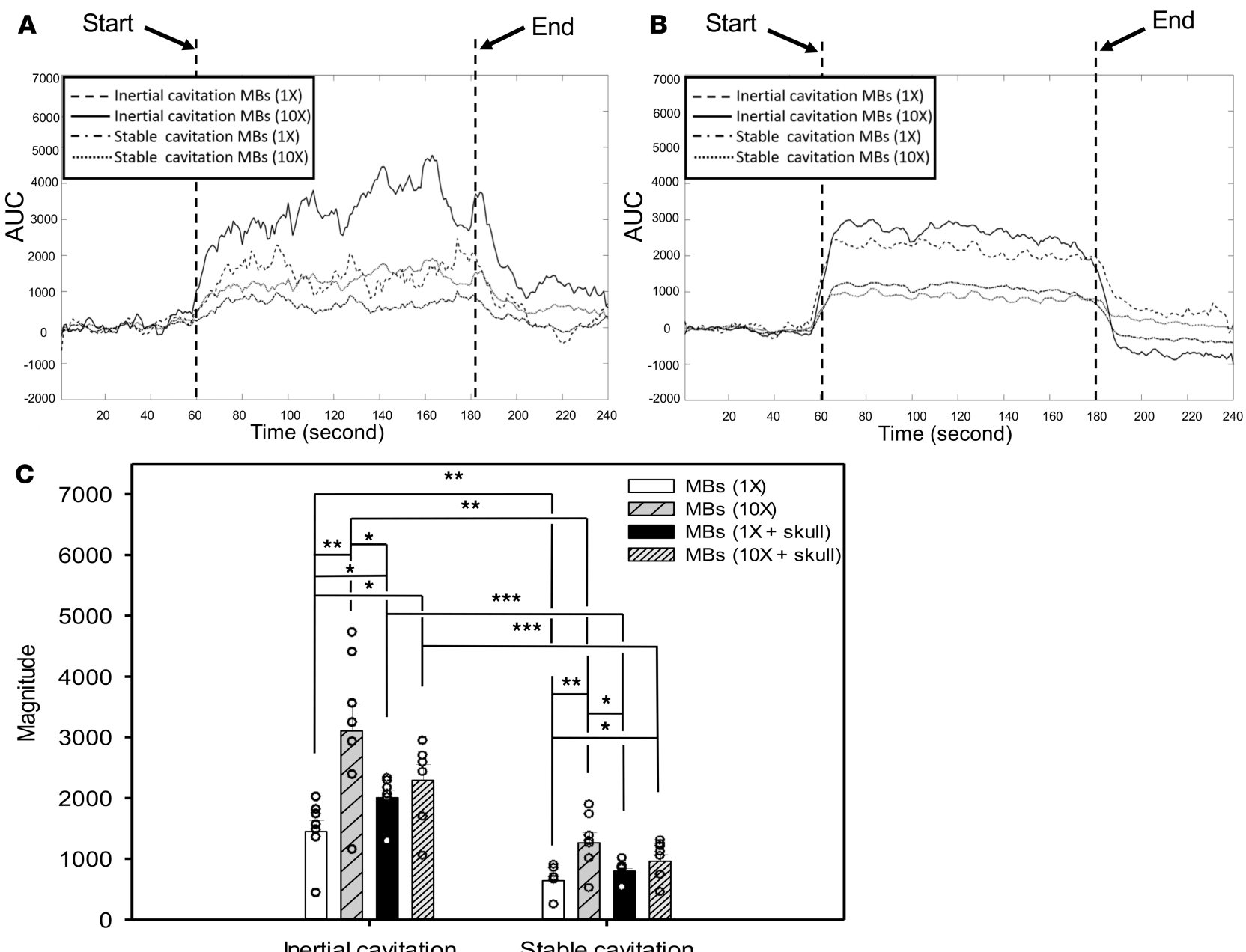

Figure 3. In vitro cavitation detection of $\mathbf{2}$ dilutions of MBs during US sonication. (A) Energy spectra acquired from PCD during US sonication for 2 dilutions of MBs. (B) Energy spectra acquired from PCD during US sonication through the skull bone for 2 dilutions of MBs. (C) Quantification of the energy density of stable cavitation and inertial cavitation in $\mathbf{A}$ and $\mathbf{B}$ ( $n=7$ per group). Data are mean \pm SD values. PCD, passive cavitation detection; US, ultrasound; MBs, microbubbles. ${ }^{*} P<0.05,{ }^{* *} P<0.01,{ }^{* * *} P<0.001 ; 2$-tailed Student's $t$ test.

US sonication was performed via the transcanal approach. The RWS group only received the injection of the mixture. The control group only received the injection of gentamicin solution without MBs. Two days after the exposure to USMBs, the cochlear and vestibular tissue was harvested for analyzing the uptake of gentamicin. In parallel with phalloidin, myosin 7a, a hair cell marker, was also used to label the hair cells to determine gentamicin uptake of the hair cells in these groups. There was a marked disparity in the distribution of gentamicin between these 2 groups (Figure 5). The USM group exhibited robust fluorescence of gentamicin labeling in the inner and outer hair cells in all turns of the cochlea - especially in the basal turn. In contrast, the RWS and control groups presented with weak and sparse gentamicin labeling in the basal and second turns, and no fluorescence was detected in the third turn. Compared with the RWS and control groups, the USM group exhibited greater uptake of gentamicin in the hair cells in all turns of the cochlea (Figure 5). In utricle and saccule of the vestibula, the USM group exhibited the most intense fluorescence of gentamicin labeling in the hair cells. The RWS and control groups presented with scattered labeling (Figure 6). These results demonstrate that transcanal USMBs enhance the uptake of gentamicin by hair cells and suggest that this technique can be applied to facilitate the administration of drugs delivered into the inner ear through the RWM.

Subsequently, a longer-term analysis of hair cell loss in the cochlea and vestibule was performed to follow up on the enhancement of gentamicin delivery by USMBs. Two weeks after the gentamicin treatment, the cochlear and vestibular tissues were harvested for analysis of the hair cell loss. The number of myosin $7 \mathrm{a}$-positive cells in the inner and outer hair cell regions was smaller in the USM group than in 

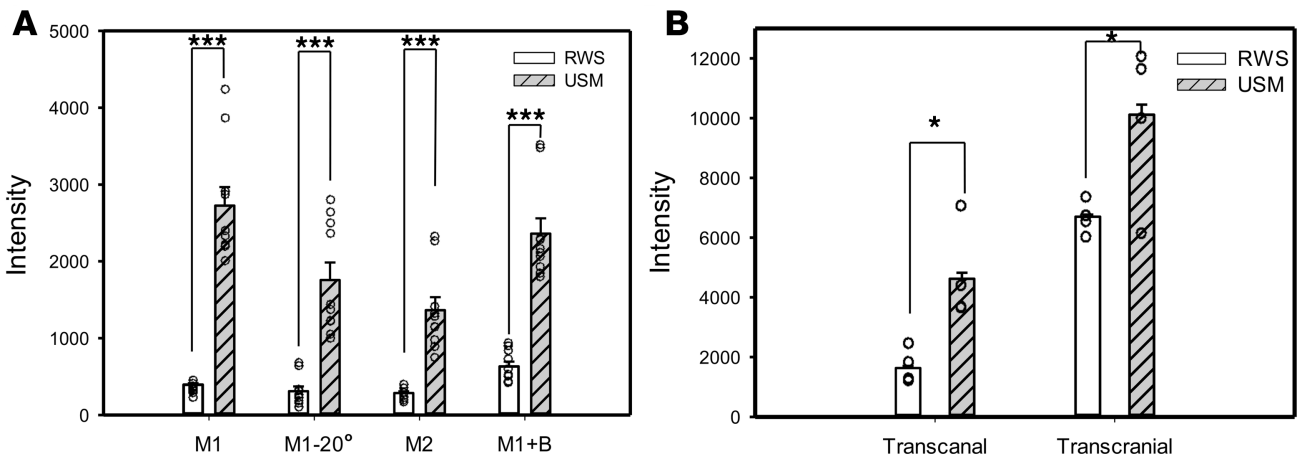

Figure 4. In vitro and in vivo efficacy of USMBs for RWM permeation. (A) In vitro biotin-FITC concentrations in the 4 experimental groups (M1, M1-20 , M2, and M1+B) with the 3D-printed diffusion apparatus $(n=10)$. (B) In vivo biotin-FITC concentrations in the perilymphatic fluid of the inner ear of the USM and RWS groups in the transcanal and transcranial arms $(n=4) .{ }^{*} P<0.05,{ }^{* *} P<0.001 ; 2$-tailed Student's $t$ test. Data are mean \pm SD values.

the RWS group (Figure 7). The basal turn of the USM group showed the severest hair cell loss. In the utricle and saccule, the USM group exhibited a drastically reduced density of myosin 7a-positive cells and severe damage to the stereocilia bundles in the sensory epithelium (Figure 8), whereas the RWS group presented a greater density of myosin 7a-positive cells and less loss of stereocilia bundles in the sensory epithelium. These results demonstrate that USMB-assisted gentamicin treatment is more effective for vestibular ablation than is the current clinical practice. Therefore, USMB-assisted drug delivery may represent an option for treatment of patients with Ménière's disease.

Finally, the presence of any auditory compromise related to this technique was investigated in order to assess its safety. The integrity of the tympanic membrane following exposure to USMB was inspected using an operating microscope. No perforation of the tympanic membrane was found in the USM group at 4 weeks after the US sonication. The loss of hair cells in the surface preparations of the organ of Corti was also investigated after exposure to USMB (Figure 9A). The results indicated that there was no significant hair cell loss in any of the 3 turns of the cochlea at 4 weeks following the exposure. Functional assessments of hearing showed no significant difference in the auditory brainstem response (ABR) threshold at any of the tested frequencies after exposure to USMBs in the USM group (Figure 9B), as well as no differences in ABR thresholds between the USM and RWS groups. These data suggest that the transcanal approach for exposure to USMB does not cause either structural or functional damage in the inner ear.

\section{Discussion}

The clinical application of using USMB cavitation to promote inner ear drug delivery requires evaluations of the sonoporation mechanisms, US parameters, and the delivery methods for US sonication and MBs. Intratympanic injection is currently the most popular method for delivering drugs into the inner ear, since it can be delivered without encountering the blood-labyrinth barrier or systemic side effects and has been used in clinical applications for many years (22). In the present study, MBs mixed with a fluorescent drug were delivered into the middle ear cavity via a transtympanic injection, and US was then applied to the temporal bone of the skull (transcranial approach) or the external auditory canal (transcanal approach) to induce the cavitation of MBs and increase the permeability of the RWM in order to enhance drug delivery. PCD was performed either directly or through the skull bone to quantify the stable cavitation and inertial cavitation.

Figure 3 shows that the average AUCs for the inertial cavitation of MBs at dilutions of $10 \times\left(1.40 \times 10^{7}\right.$ $\mathrm{MBs} / \mathrm{mL})$ and $1 \times\left(1.40 \times 10^{8} \mathrm{MBs} / \mathrm{mL}\right)$ were significantly higher than those for stable cavitation, irrespective of whether or not the US sonication was applied through the skull bone $(P<0.01)$. Moreover, the $10 \times$ dilution of MBs provided the optimal concentration for inertial USMB cavitation. These results are consistent with the results shown in Figures 1 and 2. Although the destruction efficiencies of MBs under US sonication applied through the skull bone at power densities of 3 and $4 \mathrm{~W} / \mathrm{cm}^{2}$ reached $79.1 \%-79.6 \%$, the duration of sonication was 3 minutes, which was longer than the sonication duration (1 minute) in the group with US sonication applied through the tympanic membrane. We found that sonication at a power density of $3 \mathrm{~W} / \mathrm{cm}^{2}$ for 1 minute in the transcanal approach and 3 minutes in the transcranial approach can disrupt most of the MBs and induce inertial cavitation more effectively; therefore, these parameters and conditions were used in the 

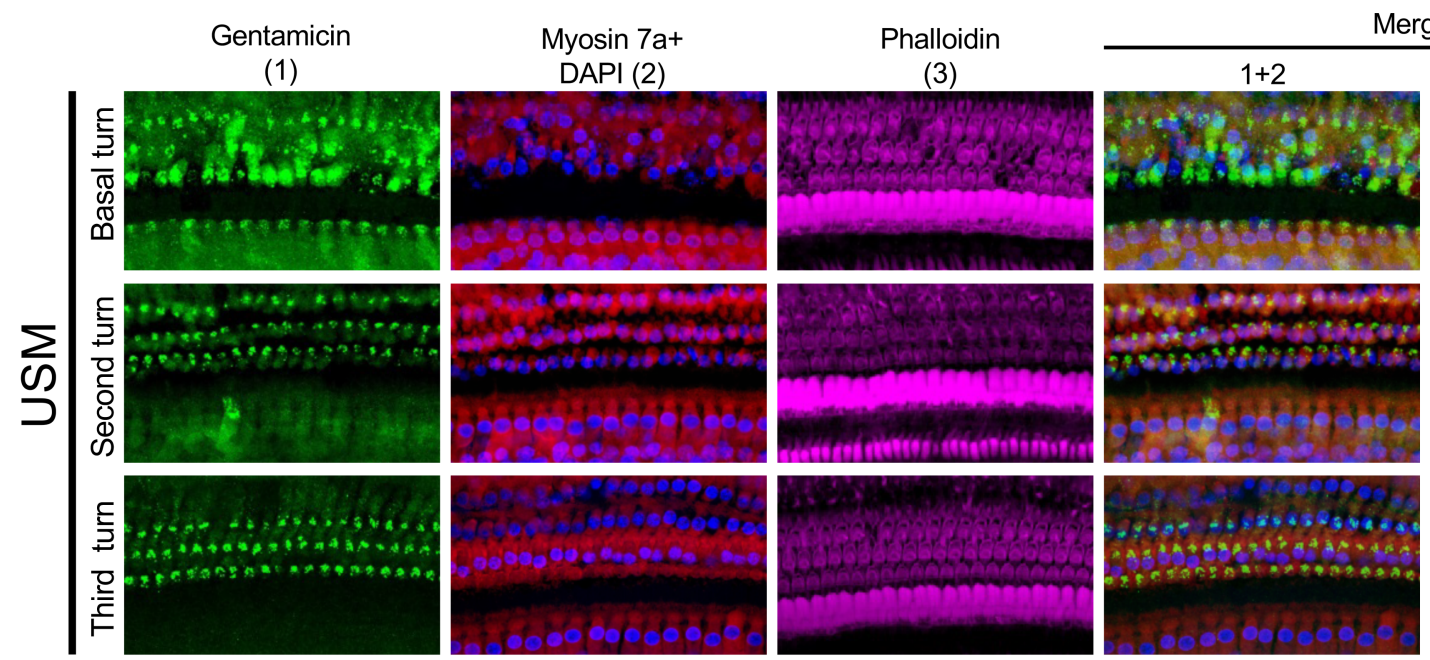

Merged
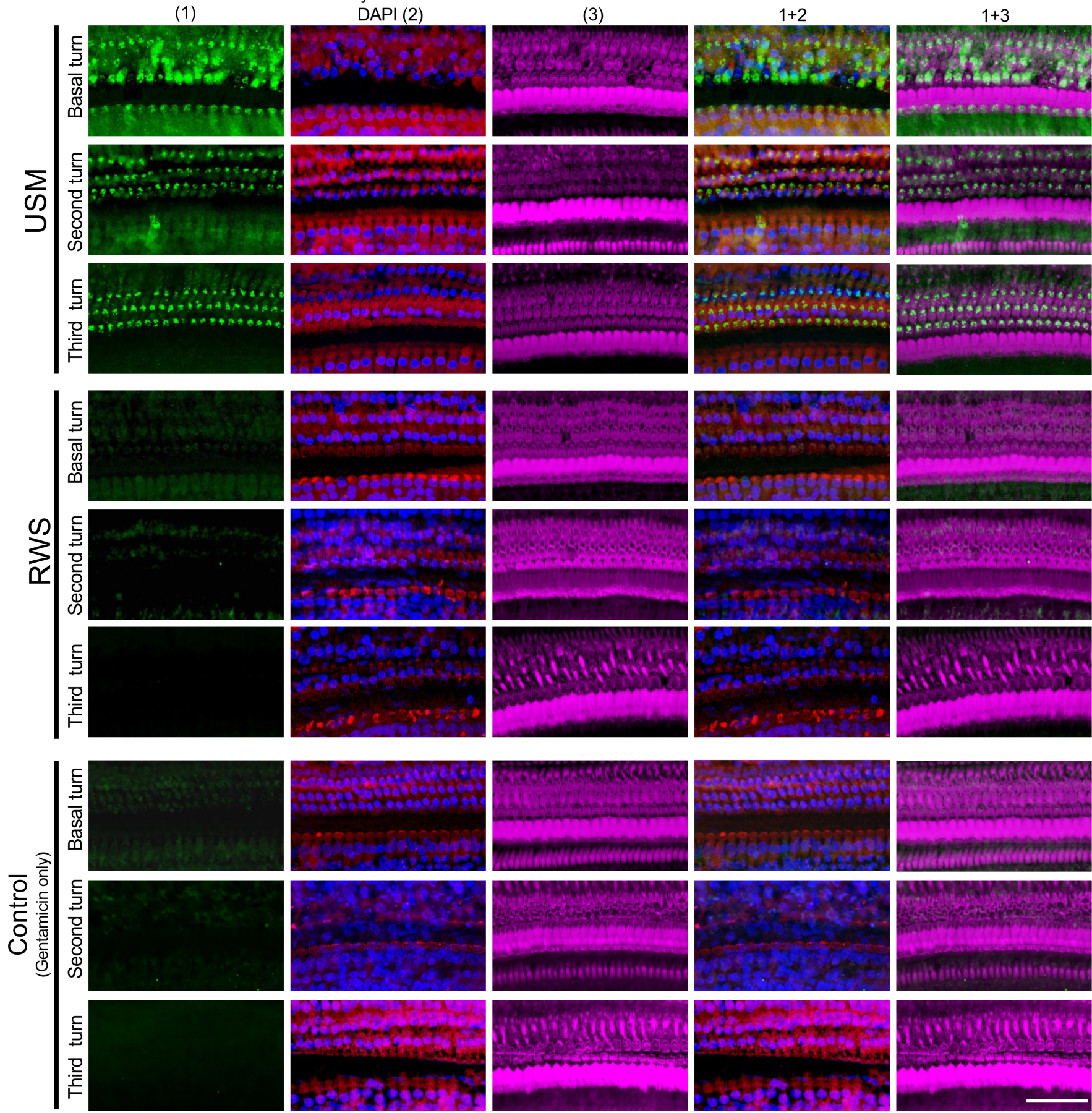

Figure 5. Exposure to USMBs enhances the cellular uptake of gentamicin in the cochlea. Samples were obtained from the cochleae 2 days after gentamicin treatments. Representative confocal microscopic images of indirect immunofluorescence staining show stronger localization of gentamicin uptake (green) in the USM group than in the RWS and control (gentamicin treatment without MBs) groups in the basal, second, and third turns. Four repetitions of this experiments were conducted. Myosin 7a, cell bodies (red); phalloidin, stereocilia bundles (magenta); DAPI, nuclei (blue). Scale bar: $50 \mu \mathrm{m}$. USM, ultrasound microbubble treatment; RWS, round window soaking; GM, gentamicin.

in vitro and in vivo experiments. However, thermal effects of USMB cavitation were more obvious on the surface of the cranium (temperature increase, $6.40^{\circ} \mathrm{C} \pm 0.70^{\circ} \mathrm{C}$ ) and in the chamber loaded with MBs (temperature increase, $2.04^{\circ} \mathrm{C} \pm 0.29^{\circ} \mathrm{C}$ ) in the transcranial approach than in the transcanal approach (temperature increase in the chamber, $1.75^{\circ} \mathrm{C} \pm 0.15^{\circ} \mathrm{C}$ ). The longer duration of sonication in the transcranial approach resulted in a significant temperature increase on the surface of the cranium, which may raise safety concerns 

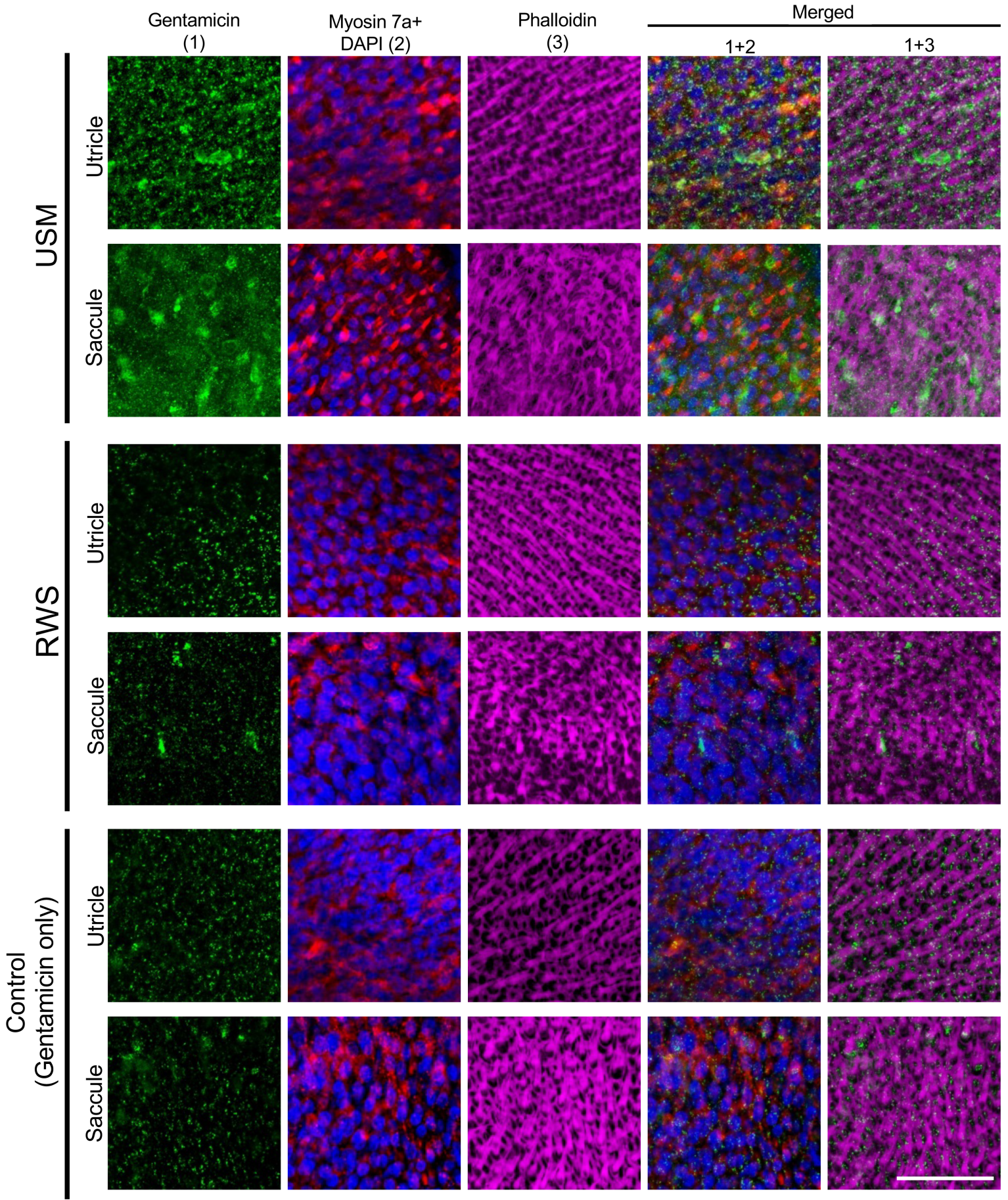

Figure 6. Exposure to USMBs enhances the cellular uptake of gentamicin in the utricle and saccule. Samples were obtained from the vestibule end organs 2 days after gentamicin treatments. Representative confocal microscopic images show the localization of gentamicin uptake (green) by indirect immunofluorescence staining. Four repetitions of this experiments were conducted. Myosin 7a, cell bodies (red); phalloidin, stereocilia bundles (magenta); DAPI, nuclei (blue). Scale bar: $50 \mu \mathrm{m}$. USM, ultrasound microbubble treatment; RWS, round window soaking; GM, gentamicin.

about any adverse events when applied for clinical trial. A temperature elevation of $1^{\circ} \mathrm{C}-1.5^{\circ} \mathrm{C}$ has been recommended to be applied indefinitely without concern on safety grounds (23). It therefore seems unlikely that the thermal adverse effects were involved in our transcanal approach.

In 1997, the US Food and Drug Administration suggested that the use of synthetic membranes, such as polysulfone or cellulose acetate, in drug-diffusion studies involving Franz cells is suitable for assessing the performance of topical formulations when biological skin is not readily available $(24,25)$. This led to several 

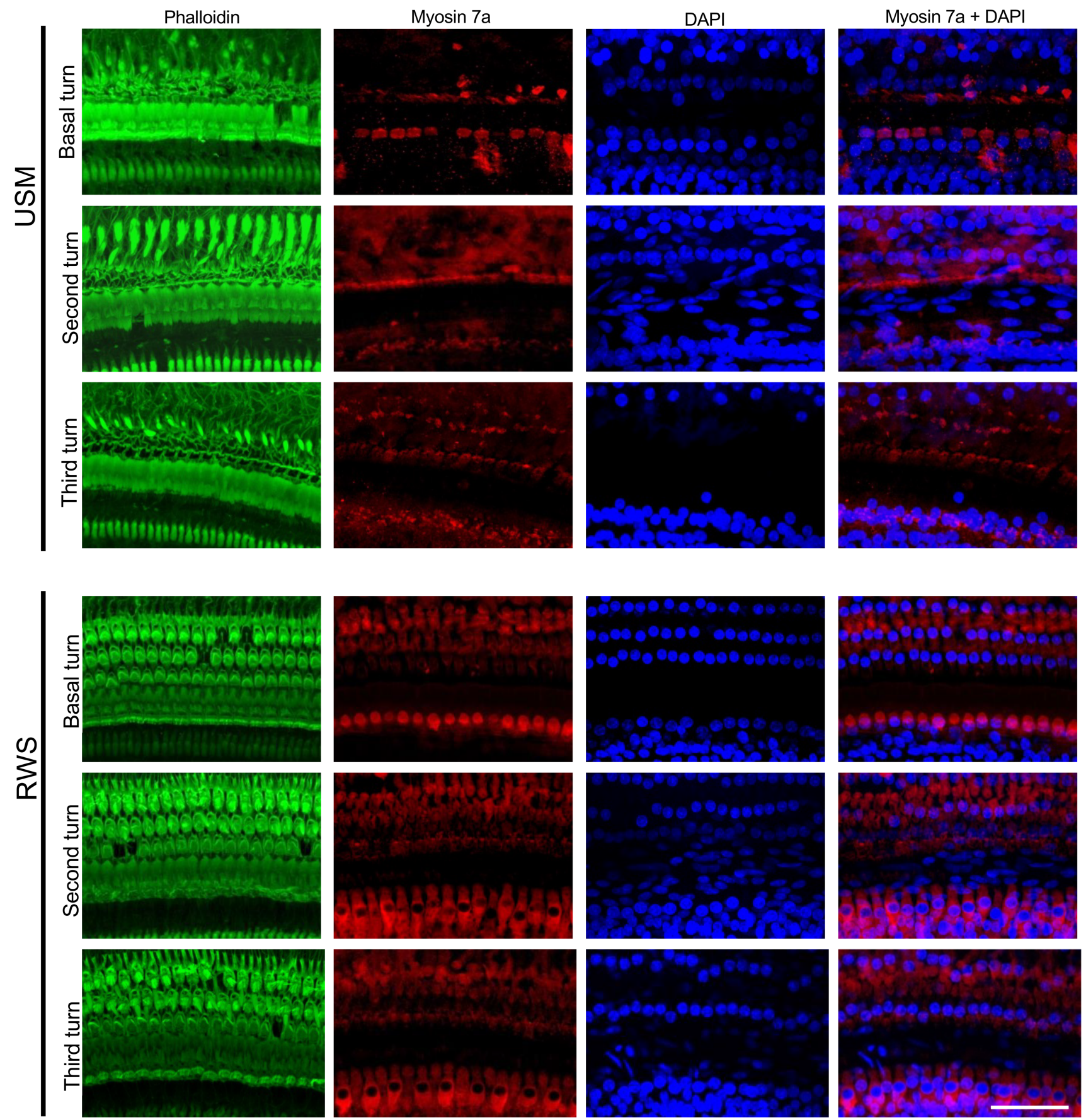

Figure 7. Exposure to USMB leads to a severer hair cell loss in the basal and second turns of the cochlea. Samples were obtained from the cochleae 2 weeks after gentamicin treatments. Representative confocal microscopic images demonstrate fewer cells stained by myosin 7a in the USM group than the RWS group in the basal turn and second turn. Four repetitions of this experiments were conducted. Phalloidin, stereocilia bundles (green); myosin 7a, cell bodies (red); DAPI, nuclei (blue). Scale bar: $50 \mu \mathrm{m}$.

studies that assessed topical drug diffusion using porous synthetic membranes $(24,26,27)$. For assessment of in vitro inner ear drug delivery, several formulations and methodologies have been investigated using a modified diffusion cell setup with a synthetic membrane (4.5-5.0 $\mu \mathrm{m}$ pore size) (28-31). For example, in a modified in vitro diffusion cell experiment, the synthetic membrane was regarded as akin to the RWM, as it separated the donor chamber above (simulating the middle ear cavity) from the receptor chamber below (simulating the inner ear) for evaluation of intratympanic formulations for inner ear delivery (28). We therefore used synthetic cellulose membranes in the present study to mimic the tympanic membrane and the 

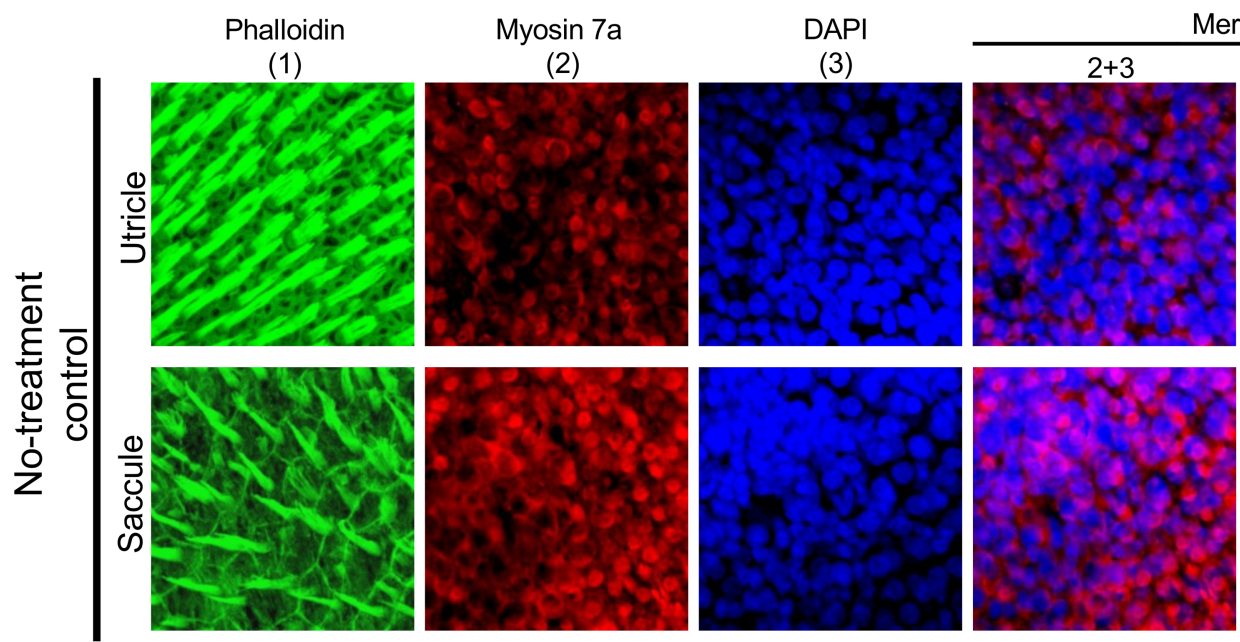

Merged
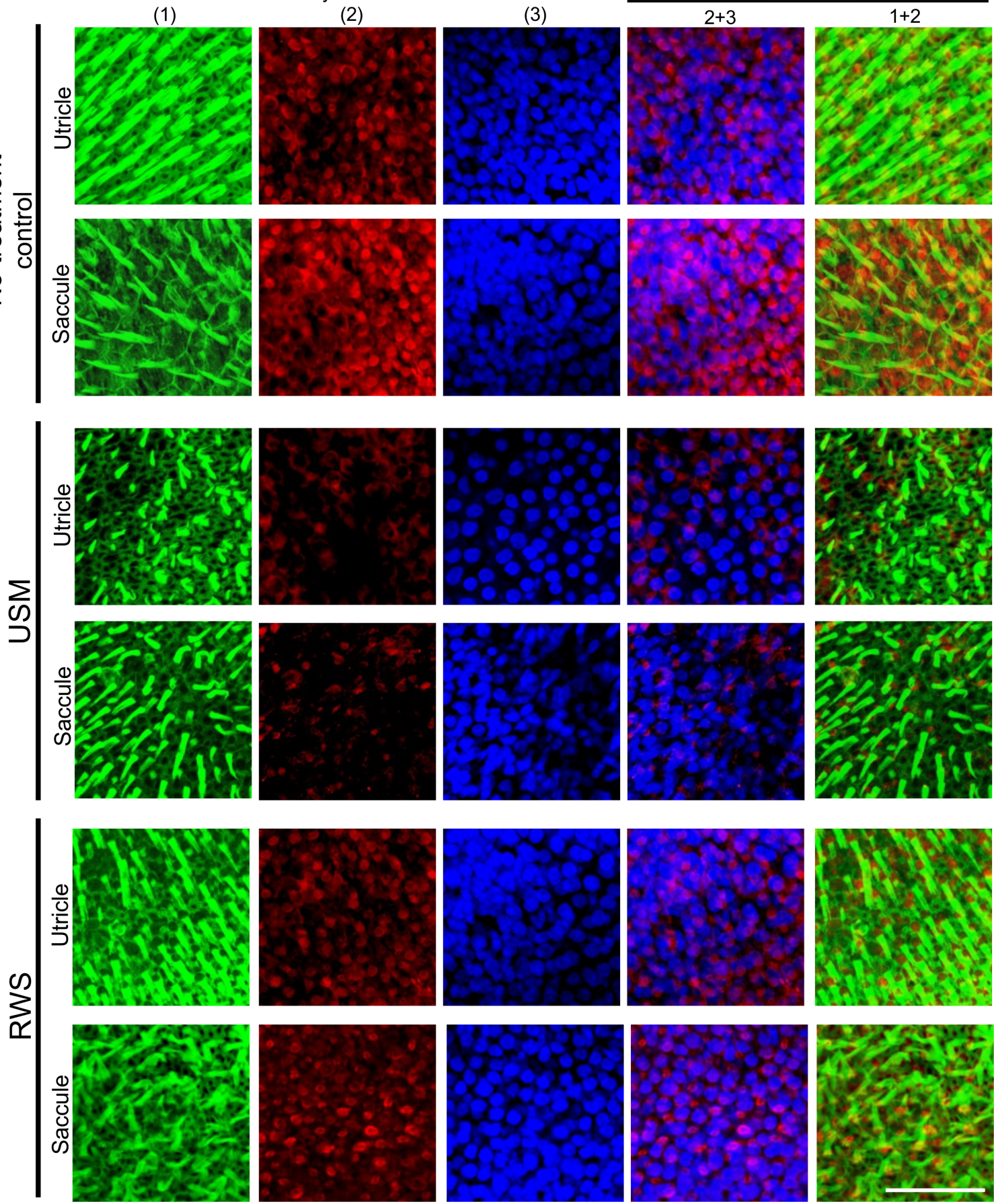

Figure 8. Exposure to USMB leads to a severer hair cell loss in the utricle and saccule of the vestibule. Samples were obtained from the vestibule 2 weeks after gentamicin treatments. Representative confocal microscopic images demonstrate that cells stained by myosin $7 a$ in the utricle and saccule from the USM group are diminished more than the RWS group. Four repetitions of this experiments were conducted. Phalloidin, stereocilia bundles (green); myosin 7a, cell bodies (red); DAPI, nuclei (blue). Scale bar: $50 \mu \mathrm{m}$.

RWM and to detect the effects of USMB on inner ear permeation. The results obtained using the 3D-printed diffusion apparatus indicated that the delivery efficiency was 4.8-fold higher in the 2-synthetic membranes model (group M2, simulating the transcanal approach) and 3.7-fold higher in the single-membrane model through the skull bone (group $\mathrm{M} 1+\mathrm{B}$, simulating the transcranial approach). Therefore, the sonication in the simulated transcanal approach at $3 \mathrm{~W} / \mathrm{cm}^{2}$ for 1 minute enhanced the drug delivery by more than $29.7 \%$ compared with that in the simulated transcranial approach (with each experiment performed 3 times). 
A No-treatment control

USM

RWS
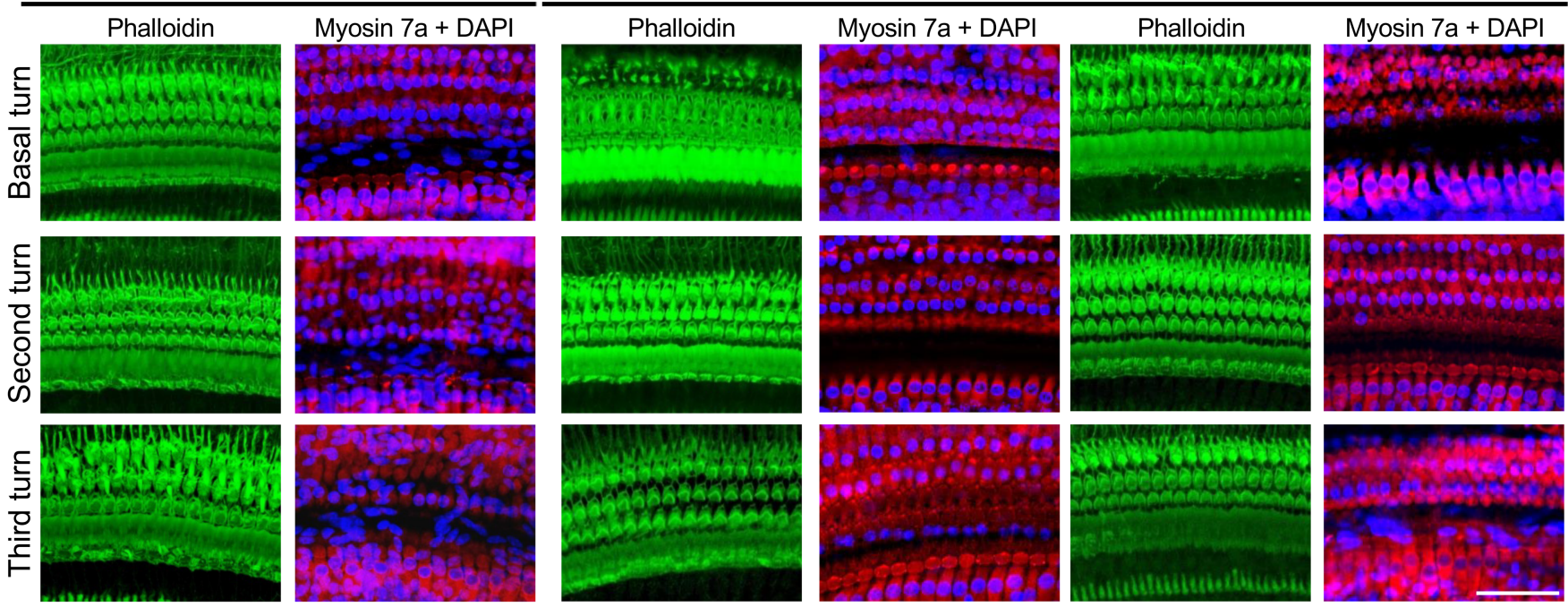

B
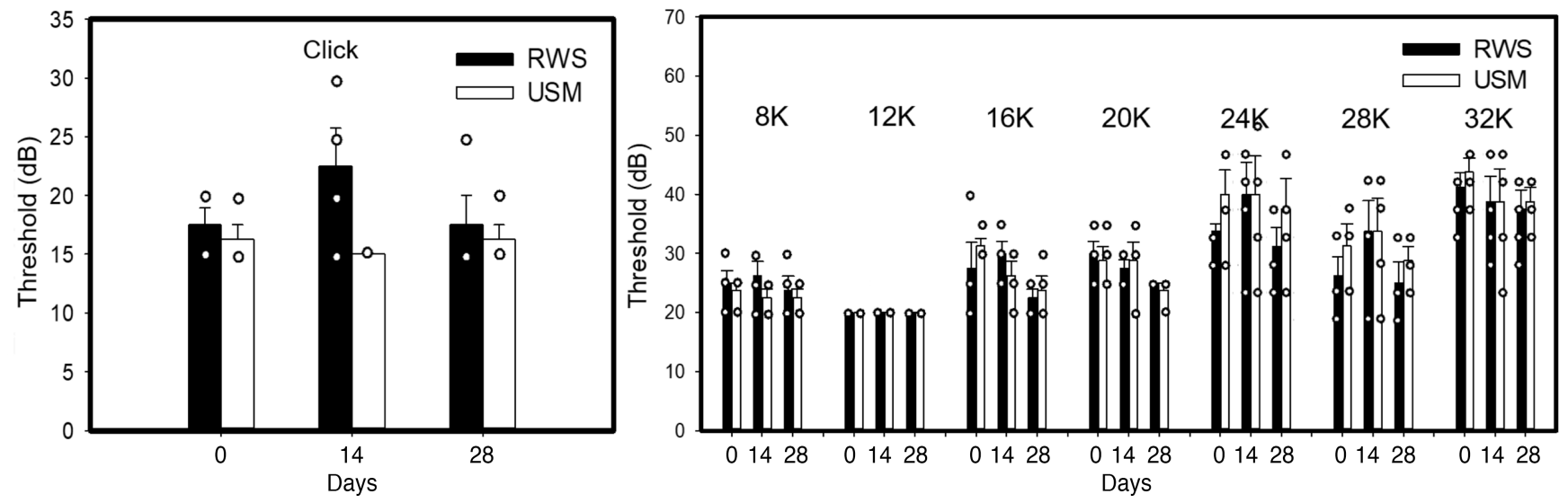

Figure 9. Hearing assessment and evaluation of the integrity of cochlear sensory epithelium in guinea pigs 28 days after ultrasound microbubble (USMB) treatments. (A) Representative images of cochlear surface preparations by immunofluorescence staining shows the nuclei (blue, DAPI), filamentous actin (green, phalloidin), and cell bodies (red, myosin 7a). Four repetitions of this experiments were conducted. Scale bar: $50 \mu \mathrm{m}$. (B) The auditory brainstem response (ABR) threshold recording with click and tone-burst stimuli before (day 0 ) and at a 1-month follow-up after USMB treatments. The results are expressed as the mean \pm SEM, with $n=4$ for each bar; 2-tailed Student's $t$ test. RWS, round window soaking; USM, ultrasound microbubble treatment.

In the small-animal experiments, the application of USMBs increased the drug delivery by approximately 2.8-fold via the transcanal approach and 1.5-fold via the transcranial approach.

Although drug delivery can be facilitated using either transcanal or transcranial exposure to USMB, the transcanal approach is superior for several reasons. First, the efficiency of delivery into the inner ear was higher for the transcanal than for the transcranial approach. Second, the middle ear cavity is very small, the structure of the ear is very intricate, and the skull bone of the guinea pig is thinner than $1 \mathrm{~mm}$ (32). By contrast, the use of the transcranial method in the human temporal bone for inner ear drug delivery is restricted by the pneumatization of the bone, which is typically $6-7 \mathrm{~mm}$ thick $(33,34)$. A third reason is that the transcanal approach is currently regarded as the ideal route for minimally invasive ear surgery (35). Compared with the retroauricular approach, the transcanal approach offers advantages that include no skin incision, no hair loss, and a short recovery time. The administration of drugs for treating diseases of the middle and inner ears is often accomplished using transtympanic injections via the external auditory canal (36). A fourth reason is that our immunofluorescence analysis of the gentamicin distribution in the cochlea by confocal microscopy showed that transcanal exposure to USMB clearly enhanced the uptake of gentamicin by the hair cells. Accordingly, transcanal exposure to USMB appears to be an effective, noninvasive method for delivering drugs to the inner ear and can be easily applied in clinical practice. Our assessments of its safety demonstrated intact hearing function and no significant hair cell loss in any of the 3 turns of the cochlea at 4 weeks following the US sonication. 


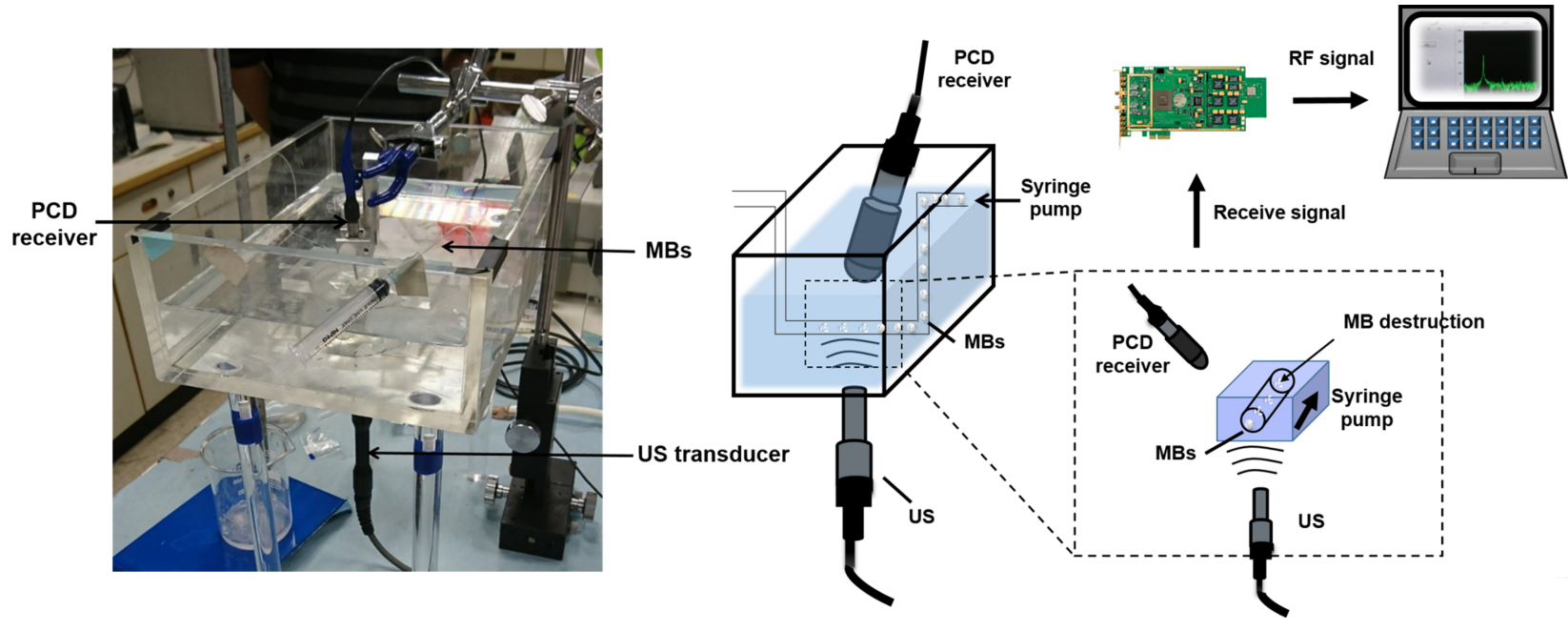

Figure 10. The passive cavitation detection (PCD) setup for measuring USMB cavitation.

Some shortcoming of the procedures applied in our study need to be overcome before translating the techniques to clinical applications. First, an intracanal incision was made in the animal in order to place the US transducer in the ear canal close to the tympanic membrane. The clinical requirement of not performing such an incision would require the size and shape of the US transducer to be customized based on the lumen of the human ear canal. Second, our technique involved repeated intratympanic injections of the mixture of drug and MBs for 3 repetitions of exposure to USMB. This is associated with a risk of persistent tympanic membrane perforation, although no perforation of the tympanic membrane was found in any of our animals at 4 weeks after exposure to USMB. A rate of $1.6 \%$ was reported for persistent perforation of the tympanic membrane following multiple intratympanic steroid injections (37). Modifying the USMB cavitation settings to reduce the number of the injections may avoid this complication and is another important issue to investigate further. Third, during exposure to USMB, the US probe was about 5 $\mathrm{mm}$ from the tympanic membrane, and this space needed to be filled with saline to ensure efficient US sonication. The US transducer should be refined so that the probe can be attached to the tympanic membrane and thereby avoid the need for water infusion into the ear canal during the US sonication of the middle ear. The design for such a facilitation of inner ear drug delivery needs to be further examined.

The presence of the blood-labyrinth barrier hampers systemic drug administration to the inner ear; therefore, the different methods and devices for enhancing local drug delivery have been devised for improving medical therapies for inner ear diseases (38). Most of these delivery devices and drug carriers are applied via the external auditory canal. The present results suggest that the application of transcanal USMB to the middle and inner ears does not damage the inner ear or auditory responses. This study is the first to our knowledge to demonstrate that the noninvasive combination of US with MBs can enhance drug delivery via the external auditory canal into the inner ear. More investigations into refining this method and its mechanisms for use in clinical applications can be expected in the near future.

In conclusion, this study investigates the feasibility of transcanal and transcranial approaches when performing USMB cavitation for enhancing the delivery of drugs to the inner ear. The PCD results indicate that the inertial cavitation of USMB is significantly higher than stable cavitation, irrespective of whether or not the US passes through the skull bone. The results obtained using the 3D-printed diffusion apparatus indicated that the delivery efficiency was higher for the transcanal USMB method than the transcranial method. This is due to the possibility that the skull bone of the guinea pigs may attenuate the US energy. The results obtained in the small-animal experiments suggest that applying USMB via a transcanal or transcranial approach targeting the middle ear would enhance drug delivery into the inner ear, with the transcanal approach exhibiting a higher delivery efficiency and shorter US sonication time. Moreover, the transcanal approach does not damage the inner ear or auditory responses. The present study shows that the use of noninvasive transcanal USMB for inner ear drug-delivery enhancement in further clinic applications can be expected. 

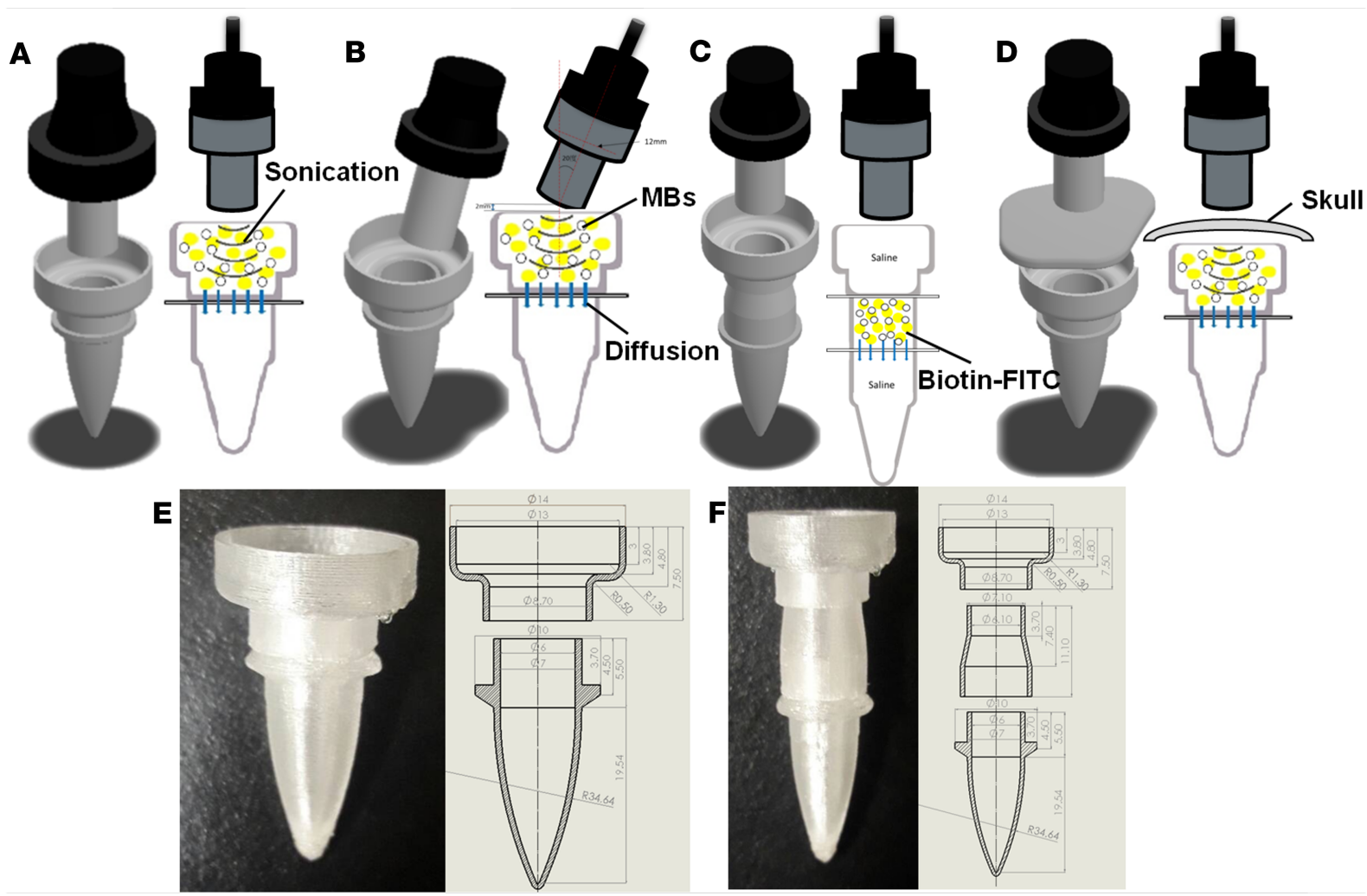

Figure 11. In vitro and in vivo efficacy of USMBs for RWM permeation. The 3D-printed apparatus mimics the pathway for US sonication passing through in vitro when the US probe is placed in the external auditory canal or on the skull, passing through the tympanic membrane and the RWM when the US probe is placed in the external auditory canal, or passing through the bone and the RWM when the probe is placed on the skull in vitro. (A) Single-membrane model (group M1). (B) US transducer deviated $20^{\circ}$ from the center in the single-membrane model (group M1-20'). (C) A 2-membrane model simulating the transcanal approach (group M2) involving placing the US probe in the external auditory canal. (D) A bony plate plus 1-membrane model simulates the transcranial approach (group M1+B), by placing the US probe on the skull. (E and F) SolidWorks designs of the custom 3D-printed single-membrane model (E) and the 2-membranes model (F).

\section{Methods}

Production of albumin-shelled MBs. Albumin-shelled MBs were prepared according to the procedure used in our previous studies $(39,40)$. Briefly, albumin-shelled MBs were generated by 2 minutes of sonication (Branson Ultrasonics) of a 10-mL solution containing $140 \mathrm{mg}$ of albumin (Octapharma) and perfluorocarbon gas in physiological saline ( $\mathrm{pH} 7.4,0.9 \%$ sodium chloride). The albumin-shelled MBs had a diameter of $1.02 \pm 0.11 \mu \mathrm{m}$ (mean $\pm \mathrm{SD}$ ) and a concentration of $1.40 \times 10^{8} \mathrm{MBs} / \mathrm{mL}$.

Optimization of US parameters for USMB cavitation by agarose phantoms. The sonication effects on MBs in vitro were evaluated by high-frequency US imaging using a commercial animal US imaging system (Prospect, S-Sharp Corporation) in tissue-mimicking agarose phantoms, as described previously (41). Briefly, a $2 \%$ agarose square-column phantom $\left(10 \times 20 \times 20 \mathrm{~mm}^{3}\right)$ was constructed with a $2 \times 2 \times 20$ $\mathrm{mm}^{3}$ chamber at its center to load $400 \mu \mathrm{L}$ of $1.4 \times 10^{7} \mathrm{MBs} / \mathrm{mL}$, followed by sonication through the tympanic membrane (which simulated transcanal USMB cavitation in the middle ear cavity) or through the skull bone (which mimicked transcranial US sonication). We used a 10-mm diameter US transducer (ST2000V, Nepagene) operating at $1 \mathrm{MHz}$. The power density was set at $1,2,3$, or $4 \mathrm{~W} / \mathrm{cm}^{2}$ with a duty cycle of $50 \%$ and a pulse repetition period of $250 \mathrm{~ms}$ for 1 minute in the transcanal group and 3 minutes in the transcranial group. High-frequency US images were obtained using a transducer with a central frequency of $40 \mathrm{MHz}$, with axial and lateral resolutions of 30 and $60 \mu \mathrm{m}$, respectively (Prospect, S-Sharp Corporation). The axial and lateral fields of view were 20 and $20 \mathrm{~mm}$, respectively. Real-time B-mode imaging was performed, and the image planes were acquired with optimization of the gain and the time-gain compensation settings, which were kept constant throughout the experiments. Images were processed with custom MATLAB programs (The MathWorks) to evaluate the destruction efficiency. The region of interest was drawn over the entire MB-loaded chamber in $2 \mathrm{D}$ imaging planes by the 
A

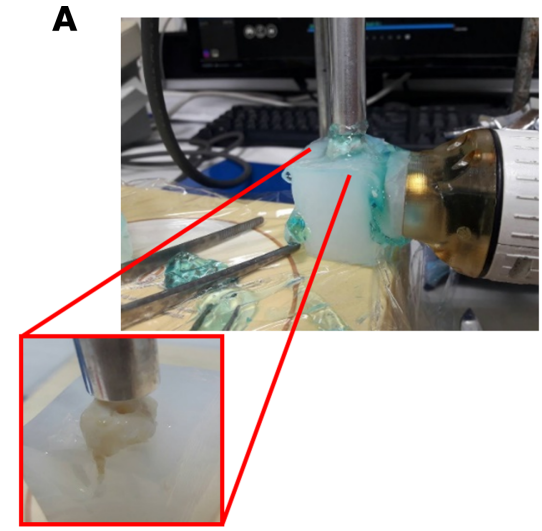

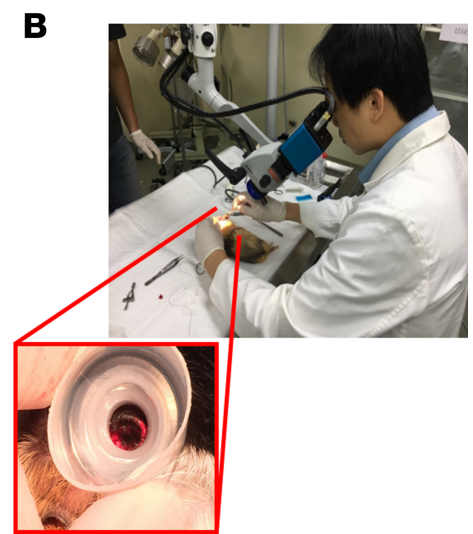

Figure 12. The ex vivo and in vivo experimental settings in the guinea pigs. (A) Photographs of the tympanic membrane taken from guinea pigs and covered on the top of a chamber (enlarged view) in the tissue-mimicking agarose phantoms. (B) Intraoperative photograph of transcanal US sonication implemented by placing the US probe in the external auditory canal, sonicating through the tympanic membrane, and causing USMB cavitation in the middle ear cavity of the guinea pigs. The tympanic membrane can be macroscopically visualized (enlarged view) with the aid of a speculum.

operator, the dynamic range was set at $50 \mathrm{~dB}$, and the average pre- and postsonication image intensities were measured in B-mode images.

$P C D$. Our previous studies have suggested that USMB disruption (i.e., inertial cavitation) is required for effective transdermal drug delivery (42-44). Recent studies have demonstrated that the acoustic-emission signals acquired in PCD could be used to quantify the subharmonic-frequency component and inertial cavitation dose, which represent the index of stable cavitation and inertial cavitation, respectively $(45,46)$. We therefore set up such a PCD system to clarify the type of cavitation that occurred in the present study (Figure 10). The transducer of a US sonoporation system and a PCD were arranged confocally using a self-made holder, with an included angle of approximately $120^{\circ}$, which was limited by their focal lengths and physical dimensions (47). The US parameters for MB cavitation in vitro were established by performing experiments at a power intensity of $3 \mathrm{~W} / \mathrm{cm}^{2}$. A polyethylene (PE) tube filled with degassed water was placed in a degassed water tank $7 \mathrm{~mm}$ below the PCD (measured from the US sonoporation transducer to the center of the PE tube). The total receiving time was 240 seconds, and $0.5 \mathrm{~mL}$ of MBs solution was injected at the 60th second. During US sonication (from 60th to 180 th second), the detector was a single-element, spherically focused $1-\mathrm{MHz}$ transducer (diameter of $12.5 \mathrm{~mm}$; V303-SU, Olympus). The holder was mounted on a computer-controlled 2D motion stage (HR8, Nanomotion). The MB echoes were received by the $1-\mathrm{MHz}$ transducer and then amplified by a broadband receiver (BR-640, Retec). The detected emissions were then quantified using spectrum-based energy-spectral-density analysis with MATLAB.

Diffusion apparatus for RWM permeation. The large volume of the Franz cells $(5-10 \mathrm{~mL})$ and the time-consuming procedures necessary to obtain transport parameters were inappropriate for the RWM permeation assay. To overcome these issues, we designed a 3D-printed diffusion apparatus to mimic the pathway for US sonication passing through the external auditory canal (the transcanal approach) or through the mastoid bone (the transcranial approach) to verify their cavitation effects for the USMB in the middle ear cavity when targeting the RWM for drug delivery in vitro. This in vitro diffusion cell apparatus was constructed using an online computer-aided-design program and produced using a 3D printer (Dforce D300, Synmao Technology Enterprise). A glycol-modified version of PE terephthalate filament is a thermoplastic material that was used to 3D print the diffusion apparatus in this study.

Two types of diffusion apparatus setups were designed with SolidWorks (Dassault Systèmes SolidWorks Corporation), as shown in Figure 11 (the 2-membranes model [group M2], simulating the transcanal approach in Figure 11, C and F, and the single-membrane model through the skull bone [group $\mathrm{M} 1+\mathrm{B}$ ], simulating the transcranial approach in Figure 11D), which consisted of a $0.5-\mathrm{mL}$ donor, an intervening cellulose membrane ( $1000 \mathrm{kDa}$; Orange Scientific), and 0.4-mL receiving compartments (Figure $11, \mathrm{~A}, \mathrm{~B}$, and $\mathrm{D}$ ); and $0.5-\mathrm{mL}$ donor compartments, an external membrane, $0.25-\mathrm{mL}$ interval chamber (the volume was consistent with the middle ear cavity), an internal membrane, and $0.4-\mathrm{mL}$ receiving 
compartments (Figure 11, C and F). Figure 11D shows the donor chamber in Figure 11, A and E, capped by a $5 \times 5 \mathrm{~mm}^{2}$ skull bone.

The drug delivery effect when the RWM was not directly sonicated by US was evaluated by deviating the US transducer $20^{\circ}$ from the center (group $\mathrm{M} 1-20^{\circ}$ ), as shown in Figure 11B. The parafilm (Pechiney Laboratory Safety Products and Apparel) occluded the outside seam of the apparatus in order to guarantee that the system was sealed. At the beginning of the experiments, each donor compartment was completely filled with the receiving medium, consisting of a $500-\mu \mathrm{L}$ mixture of biotin-FITC ( $40 \mu \mathrm{g} / \mathrm{mL}$; Anaspec) and MBs $\left(1.40 \times 10^{7} \mathrm{MBs} / \mathrm{mL}\right)$, and the probe of the sonoporation system was applied to the donor cells. The $1-\mathrm{MHz}$ US transducer of the sonoporation system positioned $5 \mathrm{~mm}$ from the top of the membrane provided sonication at a power density of $3 \mathrm{~W} / \mathrm{cm}^{2}$ (acoustic pressure, $0.266 \mathrm{MPa}$ ) for 1 minute in the single-membrane model (group M1 and group M1-20 ) and 2-membrane model (group M2), and for 3 minutes in group $\mathrm{M} 1+\mathrm{B}$. Samples obtained from the receiving compartment were analyzed by a fluorometer (Fluoroskan Ascent FL, ThermoLab Systems).

Animal study and surgical procedures. The destructive effects of the USMB through the real tympanic membrane or bony plate were examined by harvesting skull bone and the bilateral tympanic membranes with the surrounding tympanic bone from $2 \mathrm{CO}_{2}$ gas-sacrificed guinea pigs. These harvested materials were then used in an experiment of high-frequency US imaging of a tissue-mimicking agarose phantom (Figure 12A). A total of 30 pigmented male guinea pigs (weighing 250-350 g) with a normal Preyer reflex were assigned to the transcanal and transcranial arms of the experiments. Each arm was allocated to 3 groups: the USMB treatment (USM), RWS, and gentamicin only (GM) groups. Anesthesia was induced with an intramuscular injection of ketamine (Imalgene, Merial) at $80 \mathrm{mg} / \mathrm{kg}$ and xylazine (Rompun, Bayer HealthCare) at $10 \mathrm{mg} / \mathrm{kg}$, and the animals were kept warm with a heating pad for all subsequent manipulations. In the transcanal arm animals, a small incision was made in the cartilaginous portion of the external auditory canal, and a speculum was inserted into the canal to provide a better view of the tympanic membrane and the manipulation approach (Figure 12B). For USMB treatment groups, $200 \mu \mathrm{L}$ of a mixture of gentamicin $\left(40 \mathrm{mg} / \mathrm{mL}\right.$; Biological Industries) and MBs $\left(1.40 \times 10^{7}\right.$ $\mathrm{MBs} / \mathrm{mL}$ ) was injected through the antero-inferior region of the tympanic membrane into the middle ear cavity using a syringe with a 25-gauge needle. The US transducer was then inserted into the speculum and positioned $5 \mathrm{~mm}$ from the tympanic membrane. The space between the tympanic membrane and the probe was filled with saline as a conductive medium, followed by a 1-minute application of US sonication targeting the middle ear cavity at a power density of $3 \mathrm{~W} / \mathrm{cm}^{2}$ (acoustic pressure, $0.266 \mathrm{MPa}$ ). These procedures were performed under an operating microscope (F-170, Carl Zeiss). At the end of the first course of US exposure, the solution mixture in the middle ear cavity was removed and replaced with a fresh mixture of gentamicin and MBs for the second course of US exposure. Following a third course of USMB treatment, the gentamicin/MBs mixture was retained in the middle ear cavity for 2 or 14 days, according to the experimental design. For control groups, the middle ear cavity was filled as above with $200 \mu \mathrm{L}$ of a mixture of either gentamicin and MBs (for RWS group) or gentamicin and normal saline (for the GM group), and the mixture was allowed to soak the RWM for the same total duration as was used for the USM treatment. For experimental groups in the transcranial arm, the US exposure duration was extended to 3 minutes for each USMB course and a total of 3 courses was administered, as in the transcanal arm. The in vivo efficiency of USMB treatment of the RWM via the transcanal and transcranial arms was tested by replacing the mixture of gentamicin/MBs with biotin-FITC $(40 \mu \mathrm{g} / \mathrm{mL})$ and MBs $\left(1.40 \times 10^{7} \mathrm{MBs} / \mathrm{mL}\right)$, and the biotin-FITC/MBs mixture in the middle ear cavity was removed with a needle syringe at the end of the third course of USMB treatment.

After euthanizing guinea pigs with $\mathrm{CO}_{2}$ gas, the inner ear perilymph was immediately collected. The tympanic bulla was removed and a $10-\mu \mathrm{L}$ microtip on a pipette was gently inserted through a cochleostomy inferior to the RWM for perilymph aspiration. The collected samples were then centrifuged immediately, stored at $-80^{\circ} \mathrm{C}$, and later processed for fluorescence analysis.

$A B R$ recording. The auditory function of the guinea pigs was assessed by recording ABRs as described previously (48). The animals were anesthetized and kept warm with a heating pad in a sound-attenuating chamber for the duration of the recording. Subdermal needle electrodes were inserted at the vertex (positive), below the pinna of the ear (negative), and at the back (ground) of the guinea pigs. Specific stimuli (clicks and 8-, 12-, 16-, 20-, 24-, and 32-kHz tone bursts) were produced by using SigGen software (Tucker-Davis Technologies) and delivered monaurally to the external auditory canal via an insert earphone. 
The average responses from 1024 stimuli at intensities ranging from 5-90 dB sound pressure level (SPL) for each frequency were collected by decreasing the sound intensity in 5-dB steps. The resulting $A B R$ threshold was defined as the lowest intensity at which a reproducible deflection in the evoked response trace could be recognized.

Cochlear surface preparation and immunofluorescence analysis of gentamicin uptake. Guinea pigs were euthanized, and their temporal bones were quickly removed. The cochleae were fixed by the intrascalar perfusion of $4 \%$ paraformaldehyde in PBS for 1 hour at room temperature. The tissues were rinsed with PBS, and the bone surrounding the organ of Corti was removed. The organ of Corti was then carefully dissected and immersed in the same fixative overnight. The tissues were then incubated with anti-myosin 7a polyclonal antibodies (1:100; NB120-3481, Novus Biologicals) for 2 hours. After 3 washes with PBS, the tissues were incubated with Alexa Fluor 555-conjugated goat anti-rabbit antibodies (1:500; A21428, Thermo Fisher Scientific) for 1 hour. Samples were incubated with Alexa Fluor 488-conjugated phalloidin (A12379, Thermo Fisher Scientific) for 30 minutes at room temperature, rinsed with PBS, mounted in DAPI Fluoromount-G mounting medium (SouthernBiotech), and covered with a coverslip for the analysis. The flat-surface preparation of the organ of Corti was examined over its entire length. For gentamicin immunofluorescence, the fixed cochlear tissues were incubated with PBS containing 0.3\% Triton X-100 (Merck) for 30 minutes and then blocked with BlockPRO blocking buffer (Visual Protein Biotechnology) for 60 minutes at room temperature. The tissues were then incubated with anti-gentamicin monoclonal antibodies (1:100; 16101, QED Biosciences) and anti-myosin 7a polyclonal antibodies (1:100; NB120-3481, Novus Biologicals) for 2 hours. After 3 washes with PBS, the tissues were incubated with Alexa Fluor 488-conjugated donkey anti-mouse antibodies (1:200; A21202, Thermo Fisher Scientific) and Alexa Fluor 555-conjugated goat anti-rabbit antibodies (1:500; A21428, Thermo Fisher Scientific) for 1 hour. Then, samples were incubated with Alexa Fluor 633-conjugated phalloidin (1:100; A22284, Thermo Fisher Scientific) for 30 minutes at room temperature, rinsed with PBS, mounted in DAPI Fluoromount-G mounting medium, and covered with a coverslip. The fluorescence images were obtained using a confocal laser scanning microscope (Zeiss LSM 880, Carl Zeiss).

Statistics. The obtained data were analyzed statistically using 2-tailed Student's $t$ test. Different groups were compared using 1-way ANOVA, followed by Tukey's multiple-comparisons test. $P<0.05$ was considered indicative of a significant difference.

Study approval. This animal study was approved by the IACUC of the National Defense Medical Center, Taipei, Taiwan. The pigmented male guinea pigs (weighing 250-350 g) were used for experiments and cared for in accordance with protocols approved institutionally.

\section{Author contributions}

PYW, YCL, and HW performed the experiments. AHL and CPS wrote the manuscript with support from CHW. AHL, CPS, HKC, and CHW participated in planning and performing the experiments. AHL, CPS, PYW, and YCL participated in data analysis and interpretation. CPS, AHL, CHW, HLL, and HCC made substantial contributions to the conception and design of the research, data collection, and the editing of the manuscript. All of the authors have reviewed the manuscript and approved its final version.

\section{Acknowledgments}

This work was supported, in part, by grants from the Ministry of Science and Technology, Taiwan (MOST106-2221-E-011-043-MY3 to AHL, MOST107-2314-B-663-001-MY3 to CHW, and MOST1072314-B-016-027 and MOST108-2314-B-016-038 to CPS); the Tri-Service General Hospital (TSGH-C107-009 to CHW, and TSGH-C108-011 and TSGH-A-109001 to CPS); the Taichung Armed Forces General Hospital (108A14 to CHW); the National Taiwan University of Science and Technology-Tri-Service General Hospital Joint Research Program (NTUST-TSGH-108-01 to AHL); the Medical Affairs Bureau Ministry of National Defense (MAB-108-005 to CHW and MAB-109-002 to CPS); and the Teh-Tzer Study Group for Human Medical Research Foundation (A1041001-2 to CHW).

Address correspondence to: Cheng-Ping Shih, Department of Otolaryngology-Head and Neck Surgery, Tri-Service General Hospital, National Defense Medical Center, No. 325, Sec. 2, Chenggong Rd., Neihu District, Taipei City 11490, Taiwan. Phone: 886.2.8792.7192; Email: zhengping_shi@yahoo.com.tw. 
1. Salt AN, Plontke SK. Pharmacokinetic principles in the inner ear: Influence of drug properties on intratympanic applications. Hear Res. 2018;368:28-40.

2. King EB, Salt AN, Eastwood HT, O'Leary SJ. Direct entry of gadolinium into the vestibule following intratympanic applications in Guinea pigs and the influence of cochlear implantation. J Assoc Res Otolaryngol. 2011;12(6):741-751.

3. Salt AN, King EB, Hartsock JJ, Gill RM, O’Leary SJ. Marker entry into vestibular perilymph via the stapes following applications to the round window niche of guinea pigs. Hear Res. 2012;283(1-2):14-23.

4. Zhang L, Xu Y, Cao W, Xie S, Wen L, Chen G. Understanding the translocation mechanism of PLGA nanoparticles across round window membrane into the inner ear: a guideline for inner ear drug delivery based on nanomedicine. Int J Nanomedicine. 2018;13:479-492.

5. Yang KJ, et al. Optimized phospholipid-based nanoparticles for inner ear drug delivery and therapy. Biomaterials. 2018;171:133-143.

6. Wang $\mathrm{H}$, et al. Efficient cochlear gene transfection in guinea-pigs with adeno-associated viral vectors by partial digestion of round window membrane. Gene Ther. 2012;19(3):255-263.

7. Sun H, Huang A, Cao S. Current status and prospects of gene therapy for the inner ear. Hum Gene Ther. 2011;22(11):1311-1322.

8. Creber NJ, Eastwood HT, Hampson AJ, Tan J, O'Leary SJ. Adjuvant agents enhance round window membrane permeability to dexamethasone and modulate basal to apical cochlear gradients. Eur J Pharm Sci. 2019;126:69-81.

9. Juhn SK, et al. The role of inflammatory mediators in the pathogenesis of otitis media and sequelae. Clin Exp Otorhinolaryngol. 2008;1(3):117-138.

10. Kelso CM, et al. Microperforations significantly enhance diffusion across round window membrane. Otol Neurotol. 2015;36(4):694-700.

11. Stevens JP, Watanabe H, Kysar JW, Lalwani AK. Serrated needle design facilitates precise round window membrane perforation. J Biomed Mater Res A. 2016;104(7):1633-1637.

12. Sennoga CA, et al. Microbubble-mediated ultrasound drug-delivery and therapeutic monitoring. Expert Opin Drug Deliv. 2017;14(9):1031-1043.

13. Mainprize T, et al. Blood-Brain Barrier Opening in Primary Brain Tumors with Non-invasive MR-Guided Focused Ultrasound: A Clinical Safety and Feasibility Study. Sci Rep. 2019;9(1):321.

14. O'Reilly MA, Hynynen K. Blood-brain barrier: real-time feedback-controlled focused ultrasound disruption by using an acoustic emissions-based controller. Radiology. 2012;263(1):96-106.

15. Sun T, et al. Closed-loop control of targeted ultrasound drug delivery across the blood-brain/tumor barriers in a rat glioma model. Proc Natl Acad Sci USA. 2017;114(48):E10281-E10290.

16. Song KH, Harvey BK, Borden MA. State-of-the-art of microbubble-assisted blood-brain barrier disruption. Theranostics. 2018;8(16):4393-4408.

17. Shih CP, et al. Ultrasound-aided microbubbles facilitate the delivery of drugs to the inner ear via the round window membrane. J Control Release. 2013;167(2):167-174.

18. Shih CP, et al. Middle-ear dexamethasone delivery via ultrasound microbubbles attenuates noise-induced hearing loss. Laryngoscope. 2019;129(8):1907-1914.

19. Isaacson B, Nogueira JF. Endoscopic Management of Middle Ear and Temporal Bone Lesions. Otolaryngol Clin North Am. 2016;49(5):1205-1214.

20. Suarato G, Spanò R, Bertorelli R, Diaspro A, Athanassiou A, Surdo S. 3D-printed, pocket-size diffusion cells for skin permeation investigation. Proceedings. 2018;2(13):945.

21. Al-Awadi A, Olusi SO, Al-Zaid N, Prabha K. Serum B2-microglobulin concentration correlates with urinary concentrations of type 1 collagen cross-linked N-telopeptides and deoxypyridinoline in rheumatoid arthritis. Ann Saudi Med. 1998;18(2):113-116.

22. Lee KH, Ryu SH, Lee HM, Park SK, Kim HJ, Chang J. Is Intratympanic Dexamathasone Injection Effective for the Treatment of Idiopathic Sudden Sensorineural Hearing Loss? J Audiol Otol. 2015;19(3):154-158.

23. Ter Haar G. Ultrasonic imaging: safety considerations. Interface Focus. 2011;1(4):686-697.

24. Salamanca CH, Barrera-Ocampo A, Lasso JC, Camacho N, Yarce CJ. Franz Diffusion Cell Approach for Pre-Formulation Characterisation of Ketoprofen Semi-Solid Dosage Forms. Pharmaceutics. 2018;10(3):E148.

25. In vitro release testing and in vivo bioequivalence documentation. 1997. FDA-SUPAC-SS. Guidance for industry. SUPAC-SS non-sterile semisolid dosage forms. Scale-up and postapproval changes: chemistry, manufacturing and controls. https://www. fda.gov/media/71141/download.

26. Ng SF, Rouse J, Sanderson D, Eccleston G. A Comparative Study of Transmembrane Diffusion and Permeation of Ibuprofen across Synthetic Membranes Using Franz Diffusion Cells. Pharmaceutics. 2010;2(2):209-223.

27. Ng SF, Rouse JJ, Sanderson FD, Meidan V, Eccleston GM. Validation of a static Franz diffusion cell system for in vitro permeation studies. AAPS PharmSciTech. 2010;11(3):1432-1441.

28. Wazen JM, Stevens JP, Watanabe H, Kysar JW, Lalwani AK. Silver/silver chloride microneedles can detect penetration through the round window membrane. J Biomed Mater Res Part B Appl Biomater. 2017;105(2):307-311.

29. Feng L, Ward JA, Li SK, Tolia G, Hao J, Choo DI. Assessment of PLGA-PEG-PLGA copolymer hydrogel for sustained drug delivery in the ear. Curr Drug Deliv. 2014;11(2):279-286.

30. Park SH, Moon IS. Round window membrane vibration may increase the effect of intratympanic dexamethasone injection. Laryngoscope. 2014;124(6):1444-1451.

31. Liu H, Feng L, Tolia G, Liddell MR, Hao J, Li SK. Evaluation of intratympanic formulations for inner ear delivery: methodology and sustained release formulation testing. Drug Dev Ind Pharm. 2014;40(7):896-903.

32. Wysocki J. Topographical anatomy of the guinea pig temporal bone. Hear Res. 2005;199(1-2):103-110.

33. Guignard J, Arnold A, Weisstanner C, Caversaccio M, Stieger C. A Bone-Thickness Map as a Guide for Bone-Anchored Port Implantation Surgery in the Temporal Bone. Materials (Basel). 2013;6(11):5291-5301.

34. Song SW, Jun BC, Kim H, Cho Y. Evaluation of temporal bone pneumatization with growth using 3D reconstructed image of computed tomography. Auris Nasus Larynx. 2017;44(5):522-527.

35. Furukawa T, Watanabe T, Ito T, Kubota T, Kakehata S. Feasibility and advantages of transcanal endoscopic myringoplasty. Otol Neurotol. 2014;35(4):e140-e145. 
36. Light JP, Silverstein H. Transtympanic perfusion: indications and limitations. Curr Opin Otolaryngol Head Neck Surg. 2004;12(5):378-383

37. Topf MC, et al. Rate of tympanic membrane perforation after intratympanic steroid injection. Am J Otolaryngol. 2017;38(1):21-25.

38. Mäder K, Lehner E, Liebau A, Plontke SK. Controlled drug release to the inner ear: Concepts, materials, mechanisms, and per formance. Hear Res. 2018;368:49-66.

39. Liao AH, Lu YJ, Lin YC, Chen HK, Sytwu HK, Wang CH. Effectiveness of a Layer-by-Layer Microbubbles-Based Delivery System for Applying Minoxidil to Enhance Hair Growth. Theranostics. 2016;6(6):817-827.

40. Liao AH, et al. Combining Microbubble Contrast Agent with Pulsed-Laser Irradiation for Transdermal Drug Delivery. Pharmaceutics. 2018;10(4):E175.

41. Chen HK, et al. Insonation of Systemically Delivered Cisplatin-Loaded Microbubbles Significantly Attenuates Nephrotoxicity of Chemotherapy in Experimental Models of Head and Neck Cancer. Cancers (Basel). 2018;10(9):E311.

42. Oberli MA, Schoellhammer CM, Langer R, Blankschtein D. Ultrasound-enhanced transdermal delivery: recent advances and future challenges. Ther Deliv. 2014;5(7):843-857.

43. Prausnitz MR, Langer R. Transdermal drug delivery. Nat Biotechnol. 2008;26(11):1261-1268.

44. Liao AH, Hung CR, Chen HK, Chiang CP. Ultrasound-Mediated EGF-Coated-Microbubble Cavitation in Dressings for Wound-Healing Applications. Sci Rep. 2018;8(1):8327.

45. Tsai CH, Zhang JW, Liao YY, Liu HL. Real-time monitoring of focused ultrasound blood-brain barrier opening via subharmonic acoustic emission detection: implementation of confocal dual-frequency piezoelectric transducers. Phys Med Biol. 2016;61(7):2926-2946

46. Tsai CH, et al. Acoustic emission feedback planar ultrasound system for localized blood brain barrier opening monitoring. JMed Biol Eng. 2019;39(3):277-286

47. Fan $\mathrm{CH}$, et al. Submicron-bubble-enhanced focused ultrasound for blood-brain barrier disruption and improved CNS drug delivery. PLoS One. 2014;9(5):e96327.

48. Lin YS, et al. Dysregulated brain creatine kinase is associated with hearing impairment in mouse models of Huntington disease. J Clin Invest. 2011;121(4):1519-1523 